\title{
PROJECTIVE CHARACTERS OF DEGREE ONE AND THE INFLATION-RESTRICTION SEQUENCE
}

\author{
R. J. HIGGS
}

(Received 14 May 1987)

Communicated by H. Lausch

\begin{abstract}
Let $G$ be a finite group, $\alpha$ be a fixed cocycle of $G$ and $\operatorname{Proj}(G, \alpha)$ denote the set of irreducible projective characters of $G$ lying over the cocycle $\alpha$.

Suppose $N$ is a normal subgroup of $G$. Then the author shows that there exists a $G$ invariant element of $\operatorname{Proj}\left(N, \alpha_{N}\right)$ of degree 1 if and only if $[\alpha]$ is an element of the image of the inflation homomorphism from $M(G / N)$ into $M(G)$, where $M(G)$ denotes the Schur multiplier of $G$. However in many situations one can produce such $G$-invariant characters where it is not intrinsically obvious that the cocycle could be inflated. Because of this the author obtains a restatement of his original result using the Lyndon-Hochschild-Serre exact sequence of cohomology. This restatement not only resolves the apparent anomalies, but also yields as a corollary the well-known fact that the inflation-restriction sequence
\end{abstract}

$$
\{1\} \rightarrow M(G / N) \rightarrow M(G) \rightarrow M(N)
$$

is exact when $N$ is perfect.

1980 Mathematics subject classification (Amer. Math. Soc.): $20 \mathrm{C} 25$.

Keywords and phrases: projective representations of finite groups.

All groups, $G$, considered in this paper are finite and all representations of $G$ are defined over the complex numbers. The reader unfamiliar with projective representations is referred to [3] for basic definitions and elementary results.

The purpose of this paper is to investigate under which circumstances the following well-known corollary to Clifford's theorem can be generalized to projective characters.

(C) 1989 Australian Mathematical Society $0263-6115 / 89 \$ A 2.00+0.00$ 
Let $N \unlhd G$, and $\chi \in \operatorname{Irr}(G)$ such that $\left[\chi_{N}, 1_{N}\right] \neq 0$, where $1_{N}$ denotes the trivial character of $N$. Then $N \leq \operatorname{Ker}(\chi)$.

Our generalization will take the form of answering the following question.

Let $\alpha$ be a cocycle of $G$ and $N \unlhd G$. Under which necessary and sufficient conditions does there exist a $G$-invariant projective character of $N$ with degree 1 and cocycle $\alpha_{N}$ ?

Our motivation for investigating this problem is provided by Haggarty and Humphreys [1] who said "Given a subgroup $L$ of $G$, a cocycle $\alpha$ of $G$ determines a cocycle $\alpha_{L}$ of $L$ by restriction. However elements which are $\alpha_{L}$-regular in $L$ need not be $\alpha$-regular in $G$. This fact complicates the theory of projective characters."

The implication here can be taken to be that given that every element of $L$ is $\alpha$-regular (see 1.1 for this definition), then the theory of projective characters is similar to ordinary character theory. Indeed as the theory applies to induction from $L$ to $G$ this is the case, however we shall show in Section 1 that this is certainly not the case when looking at the restriction from $G$ to $L$, where $L \unlhd G$.

\section{Projective characters of degree one}

To avoid repetition we fix the following notation for the rest of this paper. Let $G$ be a group, $\alpha$ be a cocycle of $G$, and $\operatorname{Proj}(G, \alpha)$ denote the set of irreducible projective characters of $G$ with cocycle $\alpha$. We shall also use without further reference the fact due to Schur that $o([\alpha])$ in $M(G)$ divides $\xi(1)$ for all $\xi \in$ $\operatorname{Proj}(G, \alpha)$, where $M(G)$ denotes the Schur multiplier of $G$. We thus have that there is an element of $\operatorname{Proj}(G, \alpha)$ of degree 1 if and only if $[\alpha]=[1]$. We now recall some more well-known facts about projective characters in a series of lemmas. We start however with a basic definition.

DEFINITION 1.1. An element, $x$, of $G$ is said to be $\alpha$-regular if $\alpha(x, g)=$ $\alpha(g, x)$ for all $g \in C_{G}(x)$.

It is easy to check that if $[\alpha]=[\beta]$, then $x$ is $\alpha$-regular if and only if $x$ is $\beta$-regular. Also any conjugate of an $\alpha$-regular element is $\alpha$-regular, so that we may speak of the $\alpha$-regular conjugacy classes of $G$.

LEMMA 1.2. (i) There exists $\beta \in[\alpha]$ such that

$$
\frac{\beta(g, x) \beta\left(g x, g^{-1}\right)}{\beta\left(g, g^{-1}\right)}=1
$$

for all $\beta$-regular $x \in G$, and all $g \in G$. 
(ii) An element, $x$, of $G$ is $\alpha$-regular if and only if there exists $\xi \in \operatorname{Proj}(G, \alpha)$ such that $\xi(x) \neq 0$.

PROOF. See (7.2.4) and (7.2.5) of [3].

We call a cocycle satisfying the condition of $1.2(i)$ a class-function cocycle, in the sense that by (ii) the elements of $\operatorname{Proj}(G, \beta)$ are then class functions.

Let $N \unlhd G$, for $\varsigma \in \operatorname{Proj}\left(N, \alpha_{N}\right)$ we define the $g$-conjugate, $\varsigma^{g}$, of $\varsigma$ by

$$
\varsigma^{g}(x)=f_{\alpha}(g, x) \varsigma\left(g x g^{-1}\right)
$$

for $g \in G$, and all $x \in N$; where $f_{\alpha}(g, x)=\alpha(g, x) \alpha\left(g x, g^{-1}\right) / \alpha\left(g, g^{-1}\right)$. This defines an action of $G$ on $\operatorname{Proj}\left(N, \alpha_{N}\right)$ for which Clifford's theorem holds. Having defined our action we can now being to look at the relationship between $\alpha_{N^{-}}$ regular elements of $N$ and $\operatorname{Proj}\left(N, \alpha_{N}\right)$.

LEMMA 1.3. Let $N \unlhd G$ and $x$ be an $\alpha_{N}$-regular element of $N$. Then for all $g \in G, x^{g}$ is $\alpha_{N}$-regular.

Proof. Let $\zeta \in \operatorname{Proj}\left(N, \alpha_{N}\right)$ such that $\zeta(x) \neq 0$, and $g \in G$. Then $\varsigma^{g} \in$ $\operatorname{Proj}\left(N, \alpha_{N}\right)$ and $\varsigma^{g}\left(x^{g}\right)=c \zeta(x)$ for some $c \neq 0$. Thus $x^{g}$ is $\alpha_{N}$-regular.

Our next result shows that $G$-invariance, not surprisingly, does not depend upon the choice of cocycle from $[\alpha]$.

LEMMA 1.4. Let $N \unlhd G, \mu: G \rightarrow \mathbb{C}^{*}$ be a mapping with $\mu(1)=1$, and

$$
\beta(g, h)=\frac{\mu(g) \mu(h)}{\mu(g h)} \alpha(g, h) \quad \text { for all } g, h \in G .
$$

Suppose $\operatorname{Proj}\left(N, \alpha_{N}\right)=\left\{\varsigma_{1}, \ldots, \zeta_{t}\right\}$. Then

(i) $\operatorname{Proj}\left(N, \beta_{N}\right)=\left\{\mu_{N} \varsigma_{1}, \ldots, \mu_{N} \zeta_{t}\right\}$;

(ii) for $g \in G, \zeta_{i}^{g}=\varsigma_{j}$ if and only if $\left(\mu_{N} \zeta_{i}\right)^{g}=\mu_{N} \zeta_{j}$.

ProOF. (i) See pages 72-73 of [3].

(ii) Let $g \in G$. Then for all $x \in N$,

$$
\begin{aligned}
\left(\mu \zeta_{i}\right)^{g}(x) & =f_{\beta}(g, x)\left(\mu \zeta_{i}\right)\left(g x g^{-1}\right) \\
& =\frac{\beta(g, x) \beta\left(g x, g^{-1}\right)}{\beta\left(g, g^{-1}\right)} \mu\left(g x g^{-1}\right) \zeta_{i}\left(g x g^{-1}\right) \\
& =f_{\alpha}(g, x) \mu(x) \zeta_{i}\left(g x g^{-1}\right)=\mu(x) \zeta_{i}^{g}(x) .
\end{aligned}
$$

Proposition 1.5. Let $N \unlhd G$, and let inf denote the inflation homomorphism from $M(G / N)$ into $M(G)$. Then there exists a $G$-invariant $\delta \in$ $\operatorname{Proj}\left(N, \alpha_{N}\right)$ with $\delta(1)=1$ if and only if $[\alpha] \in \operatorname{Im}(\inf )$. 
Proof. Suppose there exists a $G$-invariant $\delta \in \operatorname{Proj}\left(N, \alpha_{N}\right)$ with $\delta(1)=1$. Let $P$ be an irreducible projective representation of $G$ with cocycle $\alpha$ which has $\delta$ as a constitutent of $P_{N}$. Then, if $P$ has degree $n$, we have that $P$ induces a homomorphism $\bar{P}: G / N \rightarrow P G L(n, \mathbb{C})$ defined by $\bar{P}(g N)=\Pi(P(g))$ for all $g \in$ $G$, where $\Pi$ denotes the natural homomorphism from $G L(n, \mathbb{C})$ onto $\operatorname{PGL}(n, \mathbb{C})$. Now any section of $\bar{P}$ will be a projective representation of $G / N$ with some cocycle $\beta$ of $G / N$, moreover $[\beta]$ does not depend on the choice of section, and it is clear that $\inf ([\beta])=[\alpha]$.

Conversely, suppose $[\alpha]=\inf ([\beta])$ for some $[\beta] \in M(G / N)$. Then regarding $\beta$ as a cocycle of $G$ we have that the trivial character, $1_{N}$, of $N$ is an element of $\operatorname{Proj}\left(N, \beta_{N}\right)$. As such $1_{N}$ is $G$-invariant since $\beta$ is constant on the cosets of $N$ in $G$, and hence there exists a $G$-invariant $\delta \in \operatorname{Proj}\left(N, \alpha_{N}\right)$ with $\delta(1)=1$ by 1.4 .

This result would appear to have easily answered our original question. However we shall now demonstrate that in certain circumstances it is possible to produce $G$-invariant projective characters of degree 1 in situations where it is not intrinsically obvious that the cocycle could be inflated.

LEMMA 1.6. Let $N \unlhd G$ such that $\left[\alpha_{N}\right]=[1]$. Then there exists $\delta \in$ $\operatorname{Proj}\left(N^{\prime}, \alpha_{N^{\prime}}\right)$ with $\delta(1)=1$ such that $\delta$ is $G$-invariant.

ProOF. Since $\left[\alpha_{N}\right]=[1], \mathscr{A}=\left\{\delta \in \operatorname{Proj}\left(N, \alpha_{N}\right): \delta(1)=1\right\}$ is non-empty and $G$ acts upon it. Now for $\delta \in \mathscr{A}$ we have that $\delta_{N^{\prime}}$ is irreducible, and so by Clifford's theorem there exists a bijection from $\operatorname{Irr}\left(N / N^{\prime}\right)$ onto $\mathscr{A}$ defined by $\lambda \mapsto \lambda \delta$. Now if $\xi \in \operatorname{Proj}(G, \alpha)$ such that $\delta$ is a constitutent of $\xi_{N}$, we have that $\xi_{N}=e\left(\delta_{1}+\cdots+\delta_{t}\right)$, where $\delta=\delta_{1}, \ldots, \delta_{t}$ are the distinct $G$-conjugates of $\delta$. Thus $\xi_{N^{\prime}}=e t \delta_{N^{\prime}}$, and so $\delta_{N^{\prime}}$ is $G$-invariant.

It is obvious that if there is a $G$-invariant element of $\operatorname{Proj}\left(N, \alpha_{N}\right)$ of degree 1 , then necessarily every element of $N$ is $\alpha$-regular. One could conjecture, falsely as it happens, that this was also a sufficient condition, but our next major result shows that to some extent this conjecture would be justified.

LEMMA 1.7. Let $N \unlhd G$ and $x$ be an $\alpha_{N}$-regular element of $N$. Suppose that $\alpha_{N}$ is class-function cocycle of $N$. Then for each $g \in G$ and all $y \in N$,

$$
f_{\alpha}(g, x)=f_{\alpha}\left(g, x^{y}\right) .
$$

ProOF. By 1.2 and 1.3 we may let $\varsigma \in \operatorname{Proj}\left(N, \alpha_{N}\right)$ such that $\varsigma\left(g x g^{-1}\right) \neq 0$. Now for $g \in G$ and all $y \in N$ we have that

$$
\varsigma^{g}(x)=f_{\alpha}(g, x) \varsigma\left(g x g^{-1}\right)
$$


and

$$
\begin{aligned}
\left(\varsigma^{g}\right)^{y^{-1}}(x) & =f_{\alpha}\left(y^{-1}, x\right) \varsigma^{g}\left(x^{y}\right) \\
& =\varsigma^{g}\left(x^{y}\right), \quad \text { since } \alpha_{N} \text { is a class-function cocycle; } \\
& =f_{\alpha}\left(g, x^{y}\right) \varsigma\left(g x^{y} g^{-1}\right) .
\end{aligned}
$$

But $g x g^{-1}$ and $g x^{y} g^{-1}$ are conjugate in $N$, since $G$ permutes the classes of $N$. Thus since $\alpha_{N}$ is a class-function cocycle we have that both $\varsigma\left(g x^{y} g^{-1}\right)=$ $\varsigma\left(g x g^{-1}\right) \neq 0$, and $\varsigma^{g}(x)=\varsigma^{g}\left(x^{y}\right)$, hence $f_{\alpha}(g, x)=f_{\alpha}\left(g, x^{y}\right)$.

THEOREM 1.8. Let $N \unlhd G ; \mathscr{C}_{1}, \ldots, \mathscr{C}_{r}$ be the $\alpha_{N}$-regular conjugacy classes of $N$ fixed by $g \in G$, and $x_{i} \in \mathscr{C}_{i}$. Suppose that $\alpha_{N}$ is a class-function cocycle of $N$. Then $\sum_{i=1}^{r} f_{\alpha}\left(g, x_{i}\right)$ is the number of $g$-invariant elements of $\operatorname{Proj}\left(N, \alpha_{N}\right)$.

Proof (BRAUER, ISAACS). Let $\zeta_{i}, \mathscr{C}_{i}$ for $1 \leq i \leq t$ denote respectively the elements of $\operatorname{Proj}\left(N, \alpha_{N}\right)$ and the $\alpha_{N}$-regular classes of $N$. Let $x_{i} \in \mathscr{C}_{i}$, if $\mathscr{C}_{i}^{g}=\mathscr{C}_{j}$ we shall write $x_{i}^{g}=x_{j}$. For $g \in G$, we define $A(g)=\left(a_{i j}\right)$, where $a_{i j}=1$, if $\zeta_{i}^{g}=\zeta_{j}$ and is zero otherwise. We also define $B(g)=\left(b_{i j}\right)$, where $b_{i j}=f_{\alpha}\left(g, x_{j}\right)$ if $\mathscr{C}_{i}^{g}=\mathscr{C}_{j}$ and is zero otherwise. We note from 1.7 that $f_{\alpha}\left(g, x_{j}\right)$ is independent of the choice of $x_{j} \in \mathscr{C}_{j}$. Finally let $P=\left(p_{i j}\right)$, where $p_{i j}=\zeta_{i}\left(x_{j}\right)$. Then we have that the $(l, m)$ th entry of $A(g) P$ is $\sum_{j=1}^{t} a_{l j} \zeta_{j}\left(x_{m}\right)=\varsigma_{l}^{g}\left(x_{m}\right)$; whereas the $(l, m)$ th entry of $P B(g)$ is

$$
\sum_{j=1}^{t} \varsigma_{l}\left(x_{j}\right) b_{j m}=f_{\alpha}\left(g, x_{m}\right)_{\varsigma_{l}}\left(g x_{m} g^{-1}\right)=\varsigma_{l}^{g}\left(x_{m}\right) .
$$

Thus $P^{-1} A(g) P=B(g)$ and so $\operatorname{trace}(A(g))=\operatorname{trace}(B(g))$. But trace $(A(g))$ is the number of $g$-invariant elements of $\operatorname{Proj}\left(N, \alpha_{N}\right)$, whereas $\operatorname{trace}(B(g))=$ $\sum_{i \in I} f_{\alpha}\left(g, x_{i}\right)$ where $I=\left\{i: \mathscr{C}_{i}^{g}=\mathscr{C}_{i}\right\}$.

As applications of the above theorem we have the following results.

COROLlaRY 1.9. Let $N \unlhd G$, and suppose that every element of $N$ is $\alpha$ regular. Then each $g \in G$ fixes at least one element of $\operatorname{Proj}\left(N, \alpha_{N}\right)$.

PROOF. By 1.4(ii) we may assume that $\alpha$ is a class-function cocycle of $G$. Let $g \in G$, then by 1.8 the number of $g$-invariant elements of $\operatorname{Proj}\left(N, \alpha_{N}\right)$ equals the number of classes of $N$ fixed by $g$.

COROLLARY 1.10. Let $N$ be a normal abelian subgroup of $G$ such that $G / C_{G}(N)$ is cyclic. Suppose that every element of $N$ is $\alpha$-regular. Then there exists $\delta \in \operatorname{Proj}\left(N, \alpha_{N}\right)$ with $\delta(1)=1$ which is $G$-invariant.

Proof. Let $C=C_{G}(N)$ and $\delta \in \operatorname{Proj}\left(N, \alpha_{N}\right)$. Then $\delta(1)=1$ and $C$ is a subgroup of the inertia subgroup, $I_{G}(\delta)$, of $\delta$ in $G$, since $N$ is abelian and every 
element of $N$ is $\alpha$-regular. Let $g \in G$ such that $\langle g C\rangle=G / C$, then by $1.9 g$ fixes some $\delta^{\prime} \in \operatorname{Proj}\left(N, \alpha_{N}\right)$. Thus $G=\langle g, C\rangle \leq I_{G}\left(\delta^{\prime}\right)$.

It is interesting to note that Mangold in (5.1) of [7] claimed that every element of $G$ is $\alpha$-regular if and only if $[\alpha]=[1]$. The first of the following examples demonstrates that this is in fact false in general for a non-abelian group, and hence also shows that the condition of every element of $G$ being $\alpha$-regular is not even sufficient generally, to guarantee the existence of an element of $\operatorname{Proj}(G, \alpha)$ of degree 1 .

EXAMPLES. Let $p$ be a prime number, and $H$ be the "einfachste" representation group for $\left(C_{p}\right)^{4}$ as in (3.5.4) of [3], so that $|H|=p^{10}$ and $H=$ $\left\langle x_{1}, x_{2}, x_{3}, x_{4}: x_{i}^{p}=\left[x_{i}, x_{j}, x_{k}\right]=1\right.$, for $\left.1 \leq i, j, k \leq 4\right\rangle$.

Let $s=\left[x_{1}, x_{2}\right]\left[x_{3}, x_{4}\right]$ and $A=\langle s\rangle$, so that $A \leq Z(H) \cap H^{\prime}$ and $|A|=p$. It is easy to show that no non-trivial element of $A$ is a commutator, see [5] for a generalization of this result. Now let $\lambda \in \operatorname{Irr}(A)$ be defined by $\lambda\left(s^{j}\right)=\omega^{j}$ for $\omega=e^{2 \pi i / p}$, and let $\alpha$ be the cocycle of $G_{1}=H / A$ constructed in the normal way from $\lambda$, see pages 180-182 of [2] for example. Then by construction $o([\alpha])=p$. Now with the definition and results of pages 195-197 of [2], we have that every element of $G_{1}$ is ' $\lambda$-special' trivially, and hence every element of $G_{1}$ is $\alpha$-regular.

For a different type of example let $B=\langle s, t\rangle$ where $t=\left[x_{1}, x_{3}\right], M=$ $\left\langle x_{1}, x_{3}, A\right\rangle$, and define $\mu \in \operatorname{Irr}(B)$ by $\mu\left(s^{j} t^{k}\right)=\lambda\left(s^{j}\right)$. One can then check that every element of $N=M / B$ is $\mu$-special, but that not every element of $M Z(H) / Z(H)$ is $\nu$-special for any extension, $\nu$, of $\mu$ to $Z(H)$. So if $\beta$ is the cocycle of $G_{2}=H / B$ constructed from $\mu$, we have shown that every element of the abelian group $N$ is $\beta$-regular, but that no element of $\operatorname{Proj}(N, \beta)$ can be $G_{2}$-invariant.

\section{The inflation-restriction sequence}

Let $N \unlhd G$. Then we have the Lyndon-Hochschild-Serre exact sequence of cohomology:

$$
\begin{aligned}
\{1\} \rightarrow H^{1}\left(G / N, \mathbb{C}^{*}\right) \stackrel{\inf _{1}}{\longrightarrow} H^{1}\left(G, \mathbb{C}^{*}\right) \stackrel{\text { res }_{1}}{\longrightarrow} H^{1}\left(N, \mathbb{C}^{*}\right)^{G} \\
\stackrel{\operatorname{tra}}{\longrightarrow} M(G / N) \stackrel{\text { inf }_{2}}{\longrightarrow} M(G)
\end{aligned}
$$

where the action of all groups on $C^{*}$ is trivial, see [6, page 354].

It is clear that we may replace $M(G)$ in this exact sequence by $M(G)^{\#}=$ $\left\{[\alpha] \in M(G):\left[\alpha_{N}\right]=[1]\right\}$. In this section we shall extend this new sequence one term to the right, and in doing so we shall give a practical test to see whether an element of $M(G)^{\#}$ is in the image of inf $_{2}$. Thus it is 1.5 which connects the results of Section 1 to those of this section. 
LEMMA 2.1. Let $N \unlhd G, \alpha$ be a cocycle of $G$ such that $[\alpha] \in M(G)^{\#}$, and $\delta \in \operatorname{Proj}\left(N, \alpha_{N}\right)$ with $\delta(1)=1$. Then

(i) the mapping $\alpha^{\prime}: G / N \rightarrow H^{1}\left(N, \mathbb{C}^{*}\right)$ defined by $\alpha^{\prime}(g N)=\delta / \delta^{g}$ is a crossed homomorphism;

(ii) the mapping $\tau: M(G)^{\#} \rightarrow H^{1}\left(G / N, H^{1}\left(N, \mathbb{C}^{*}\right)\right)$ defined by $\tau([\alpha])=\left[\alpha^{\prime}\right]$ is a homomorphism.

ProOF. (i) Let $g \in G$. Then $\delta^{g}, \delta \in \operatorname{Proj}\left(N, \alpha_{N}\right)$, and so since $\delta(1)=1$ we have that $\delta / \delta^{g} \in H^{1}\left(N, C^{*}\right)$. Now let $g_{1}, g_{2} \in G$, and suppose that $g_{1} x=g_{2}$ for $x \in N$. Then

$$
\alpha^{\prime}\left(g_{2} N\right)=\frac{\delta}{\delta^{g_{1} x}}=\frac{\delta}{\left(\delta^{g_{1}}\right)^{x}}=\frac{\delta}{\delta^{g_{1}}}=\alpha^{\prime}\left(g_{1} N\right),
$$

since $N \leq I_{G}\left(\delta^{g_{1}}\right)$. Thus $\alpha^{\prime}$ is well defined. Finally let $g_{1}, g_{2} \in G$. Then

$$
\alpha^{\prime}\left(g_{1} g_{2} N\right)=\frac{\delta}{\delta^{g_{1} g_{2}}}=\left(\frac{\delta}{\delta^{g_{1}}}\right)^{g_{2}} \frac{\delta}{\delta^{g_{2}}}=\left(\alpha^{\prime}\left(g_{1} N\right)\right)^{g_{2} N} \alpha^{\prime}\left(g_{2} N\right) .
$$

(ii) Suppose $\beta \in[\alpha]$, and let $\mu: G \rightarrow \mathbb{C}^{*}$ be a mapping with $\mu(1)=1$ such that

$$
\beta(g, h)=\frac{\mu(g) \mu(h)}{\mu(g h)} \alpha(g, h) \quad \text { for all } g, h \in G .
$$

Let $\nu \in \operatorname{Proj}\left(N, \beta_{N}\right)$ with $\nu(1)=1$. Then by 1.4 we have that $\nu=\mu_{N} \delta_{1}$ for some $\delta_{1} \in \operatorname{Proj}\left(N, \alpha_{N}\right)$. But $\delta_{1}=\lambda \delta$ for $\lambda \in H^{1}\left(N, C^{*}\right)$ as in the proof of 1.6. Thus

$$
\frac{\nu}{\nu^{g}}=\frac{\lambda}{\lambda^{g}} \frac{\mu_{N} \delta}{\left(\mu_{N} \delta\right)^{g}}=\frac{\lambda}{\lambda^{g}} \frac{\delta}{\delta^{g}}
$$

as in the proof of 1.4 , and so $\tau$ is well defined. Clearly $\tau$ is a homomorphism.

THEOREM 2.2. Let $N \unlhd G$. Then the sequence

$$
M(G / N) \stackrel{\text { inf }}{\rightarrow} M(G)^{\#} \stackrel{\tau}{\rightarrow} H^{1}\left(G / N, H^{1}\left(N, C^{*}\right)\right)
$$

is exact.

PROOF. By 1.5 we have that $\operatorname{Im}(\inf ) \leq \operatorname{Ker}(\tau)$. Let $[\alpha] \in \operatorname{Ker}(\tau)$. Then for $\delta \in \operatorname{Proj}\left(N, \alpha_{N}\right)$ with $\delta(1)=1$, we have that $\delta / \delta^{g}=\lambda / \lambda^{g}$ for some $\lambda \epsilon$ $H^{1}\left(N, C^{*}\right)$. But then $\delta \lambda^{-1}$ is $G$-invariant, and so by 1.5 we obtain that $[\alpha] \in$ $\operatorname{Im}(\mathrm{inf})$.

The above theorem can be regarded as a generalization of a result of Read, see (4.4.5) of [3], which deals with the special case when $N$ is a central subgroup of $G$. We now mention some applications of 2.2 , the first being well known. 
COROLlLARY 2.3. Let $N$ be a perfect normal subgroup of $G$. Then the sequence

$$
\{1\} \rightarrow M(G / N) \stackrel{\text { inf }}{\longrightarrow} M(G) \stackrel{\text { res }}{\longrightarrow} M(N)
$$

is exact.

ProOF. We start by noting that $H^{1}\left(N, \mathbb{C}^{*}\right)=\{1\}$, since $N^{\prime}=N$. Thus by 2.2 we have that $\operatorname{Ker}($ res $)=M(G)^{\#}=\operatorname{Im}($ inf $)$.

Our next result was used by Liebler and Yellen in (2.4) of [4] to help prove that groups of central type are solvable.

COROLLARY 2.4. Let $N \unlhd G$, and suppose that $\left(|G / N|,\left|N / N^{\prime}\right|\right)=1$. Then $M(G)^{\#}=\operatorname{Im}($ inf $)$.

ProOF. By the Schur-Zassenhaus theorem we have that $H^{1}\left(G / N, H^{1}\left(N, C^{*}\right)\right)$ is trivial, and so the desired result is immediate from 2.2.

COROLlary 2.5. Suppose $G$ is metacyclic, and let $N \unlhd G$ such that both $N$ and $G / N$ are cyclic. Then $M(G)$ is isomorphic to a subgroup of $H^{1}(G / N, N)$.

ProOF. From 2.2 we have that the sequence

$$
\{1\} \rightarrow M(G) \stackrel{\tau}{\rightarrow} H^{1}(G / N, \operatorname{Irr}(N))
$$

is exact, since $M(G)^{\#}=M(G)$. Thus $\tau$ is a monomorphism.

For our last application we can now explain the result of 1.6.

COROLlaRY 2.6. Let $N_{1}, N_{2} \unlhd G$ with $N_{2} \leq N_{1}, T$ denote the image of res: $H^{1}\left(N_{1}, C^{*}\right) \rightarrow H^{1}\left(N_{2}, C^{*}\right)$, and $M(G)^{\# i}=\left\{[\alpha] \in M(G):\left[\alpha_{N_{i}}\right]=\right.$ [1]\}. Then the homomorphism $\tau: M(G)^{\# 2} \rightarrow H^{1}\left(G / N_{2}, H^{1}\left(N_{2}, C^{*}\right)\right)$ defined in 2.1, induces by restriction to $M(G)^{\#_{1}}$ a homomorphism from $M(G)^{\#_{1}}$ into $H^{1}\left(G / N_{2}, T\right)$.

ProOF. Let $[\alpha] \in M(G)^{\# 1}$, and $\delta \in \operatorname{Proj}\left(N_{1}, \alpha_{N_{1}}\right)$ with $\delta(1)=1$. Then by 2.1 we have that $\tau([\alpha])=\left[\alpha^{\prime}\right]$, where $\alpha^{\prime}\left(g N_{2}\right)=\left(\frac{\delta}{\delta^{g}}\right)_{N_{2}} \in T$.

In the situation of 1.6 we have that $N_{2}=N_{1}^{\prime}$ and so $T=\{1\}$, with the above notation. Thus by 2.2 we obtain that $M(G)^{\# 1}$ is a subgroup of inf : $M\left(G / N_{1}^{\prime}\right) \rightarrow$ $M(G)$. 


\section{References}

[1] R. J. Haggarty and J. F. Humphreys, 'Projective characters of finite groups', Proc. London Math. Soc. (3) 36 (1978), 176-192.

[2] I. M. Isaacs, Character theory of finite groups (Pure and Applied Mathematics, a Series of Monographs and Textbooks 69, Academic Press, New York, London, 1976).

[3] G. Karpilovsky, Projective representations of finite groups (Monographs and Textbooks in Pure and Applied Mathematics 94, Marcel Dekker, New York, 1985).

[4] R. A. Liebler and J. E. Yellen, 'In search of nonsolvable groups of central type', Pacific J. Math. 82 (1979) 485-492.

[5] I. D. Macdonald, 'Commutators and their products', Amer. Math. Monthly 93 (1986), 440-443.

[6] S. Mac Lane, Homology (Die Grundlehren der mathematischen Wissenschafter in Einzeldarstellungen 114, Springer-Verlag, Berlin, Heidelberg, New York, 1967).

[7] Ruth Mangold, 'Beitrage zur Theorie der Darstellungen endlicher Gruppen durch Kollineationen', Mitt. Math. Sem. Giessen 69 (1966), 1-44.

Department of Mathematics

University College Dublin

Belfield

Dublin 4, Ireland 